\section{A) Check for updates}

Cite this: Food Funct., 2017, 8, 2583

\title{
Fabrication, characterisation and stability of oil-in- water emulsions stabilised by solid lipid particles: the role of particle characteristics and emulsion microstructure upon Pickering functionality
}

\author{
I. Zafeiri, (D)*a P. Smith, ${ }^{\text {b I. T. Norton }}{ }^{a}$ and F. Spyropoulos ${ }^{a}$
}

The quest to identify and use bio-based particles with a Pickering stabilisation potential for food applications has lately been particularly substantial and includes, among other candidates, lipid-based particles. The present study investigates the ability of solid lipid particles to stabilise oil-in-water (o/w) emulsions against coalescence. Results obtained showed that emulsion stability could be achieved when low amounts ( $0.8 \mathrm{wt} / \mathrm{wt} \%)$ of a surface active species (e.g. Tween 80 or NaCas) were used in particles' fabrication. Triple staining of the o/w emulsions enabled the visualisation of emulsion droplets' surface via confocal microscopy. This revealed an interfacial location of the lipid particles, hence confirming stabilisation via a Pickering mechanism. Emulsion droplet size was controlled by varying several formulation parameters, such as the type of the lipid and surface active component, the processing route and the polarity of the dispersed phase. Differential scanning calorimetry (DSC) was employed as the analytical tool to quantify the amount of crystalline material available to stabilise the emulsion droplets at different intervals during the experimental timeframe. Dissolution of lipid particles in the oil phase was observed and evolved distinctly between a wax and a triglyceride, and in the presence of a non-ionic surfactant and a protein. Yet, this behaviour did not result in emulsion destabilisation. Moreover, emulsion's thermal stability was found to be determined by the behaviour of lipid particles under temperature effects.

Received 11th April 2017, Accepted 24th June 2017 DOI: $10.1039 / \mathrm{c} 7 \mathrm{fo} 00559 \mathrm{~h}$ rsc.li/food-function oil/water interfaces within an emulsification process. By means of formation of a protective interfacial layer, emulsifiers assist in maintaining a sufficient distance between emulsion droplets, thereby hindering/preventing their aggregation/ coalescence. Typical emulsifiers are low molecular weight surfactants, phospholipids and also macromolecular species such as proteins, and polysaccharides. ${ }^{1}$

Despite their established effectiveness, there is a growing shift in the food industry towards the - partial or full - replacement of emulsifiers in emulsion-based products by - at least perceived to be as - natural, and more sustainable ingredients. $^{2,3}$ This is in line with the drive for "clean-label" food products fuelled by the growing consumers' demands for healthy, safe and at the same time as natural as possible foodstuffs. ${ }^{4}$ The most appealing candidates are undoubtedly colloidal particles which owing to their unsurpassed features, they have attracted a significant surge of interest within physical scientists, both academically and industrially. Emulsion droplets stabilised by colloidal particles (Pickering emulsions) combine the benefits of prolonged stability against coalescence, ${ }^{5,6}$ improvement in lipid oxidative stability, ${ }^{7,8}$ increased resistance to shear, ${ }^{9,10}$ and controlled release across the interface. ${ }^{11}$ 
Fat crystals (e.g. triacylglycerols (TAGs), etc.) are a significant class of solid particles that confer stability in a number of everyday food products such as margarine, whipped cream and ice cream. It has been proposed that their stabilisation mechanism is based on either the interfacial adsorption of previously formed crystals and/or originate from a threedimensional particle network formed in the continuous phase. ${ }^{12,13}$ Fat crystals with tailored properties have also been investigated for their potential to stabilise emulsions, although relevant work in the literature is relatively limited. In particular, there is a dearth of studies centered on production of lipid-based particles where emulsification and crystallisation take place as separate processes. Garti et al. prepared submicron $\alpha$-polymorph tristearin crystals via flash-crystallisation within a liquid oil, and then used these dispersions as the continuous phase of water-in-oil (w/o) emulsions. ${ }^{14}$ The obtained emulsions were prone to aggregation and flocculation, and the authors showed that sufficient amounts of an emulsifier were necessary to trigger adsorption of particles to the interface. More recent work by Pawlik et al. demonstrated a preference of solid tripalmitin particles to form oil or water continuous Pickering emulsions depending on the chemistry of the emulsifiers used during particle fabrication. ${ }^{15}$ The aqueous lipid dispersions were produced by melt-emulsification followed by crystallisation, and subsequent use as the continuous phase of emulsions. The emulsions were stable to coalescence for a storage period of 70 days.

An important point is that the amount of solid matter that provides stabilisation needs to be maintained over time if these structures are to be utilised commercially. Evidence from previous studies showed that within an emulsion, transfer of lipids between liquid droplets is taking place and this mechanism hinges on the type of lipid molecules (e.g. size and polarity), and the presence of surfactant micelles and cosolutes in the aqueous phase. ${ }^{16-19}$ Specifically for solid lipid nanoparticles (SLN), the addition of liquid oil to the aqueous suspension of SLN triggers mass transfer phenomena that result in the gradual dissolution of the particles over the course of storage time. ${ }^{20}$ The authors of that work have looked at the stability of solid lipid nanoparticles in the presence of pure liquid oils (alkanes) and suggested a dissolution mechanism which embodies solubilisation of lipid molecules from the oil droplets into the aqueous phase, diffusion and subsequent adsorption at the SLN surface, and finally SLN dissolution in the adsorbed liquid surface layer. It needs to be stressed that SLN in that case were not in contact with the emulsion droplets.

Previous work by the authors on the same systems, has shown that lipid particles, depending on the lipid source and the type of the surface active species used in their manufacturing, can be designed to own microstructural characteristics that are closely linked to Pickering functionality; i.e. size and interfacial behaviour. ${ }^{21}$ The aim of the present study is to fully assess the potential of solid lipid particles to act as Pickering stabilisers of oil-in-water $(\mathrm{o} / \mathrm{w})$ emulsions. Particles were prepared from a wax (cetyl palmitate) and a triglyceride (tristearin) source and were fabricated in the presence of Tween 80 or sodium caseinate (NaCas). Emulsion formation and subsequent stability were investigated as a function of the former formulation variables. Different dispersed phase mass fractions and production methods of varying shear levels were assessed. Emulsions produced in the presence of solid lipid particles were characterised by laser diffraction and confocal microscopy, both upon fabrication and following varying periods of storage. A specific attempt was made to link the level of solid matter and emulsion stability with the resulting microstructure over time, or as a result of temperature conditions, as well as type of the dispersed phase.

\section{Materials and methods}

\section{Materials}

Lipid particles were prepared using microcrystalline glyceryl tristearate (tristearin) (IOI Oleochemicals GmbH, Hamburg, Germany) and cetyl palmitate (Gattefossé, France). The particles were formed in the presence of two surface active species: polyoxyethylene sorbitan monooleate (Tween 80) and casein sodium salt (NaCas) derived from bovine milk (SigmaAldrich, UK).

For emulsions production, commercially available sunflower oil (dielectric constant at $20{ }^{\circ} \mathrm{C}, \varepsilon_{\mathrm{r}} \approx 3.1^{22}$ ) was used without further purification. In addition, pure alkanes $n$-dodecane and $n$-hexadecane $\left(\varepsilon_{\mathrm{r}}=2\right.$ and 2.14 respectively $\left.^{23}\right)$ (Sigma-Aldrich, UK) were also used for comparative purposes. Double distilled water from Milli-Q systems (Millipore, Watford, UK) was used throughout the study.

\section{Methods}

Preparation of oil-in-water emulsions. Oil-in-water $(\mathrm{o} / \mathrm{w})$ emulsions were prepared with a $80 \%$ (wt/wt) aqueous phase containing solid lipid particles and a $20 \%$ (wt/wt) sunflower oil phase. Lipid particle suspensions (tristearin, cetyl palmitate) were first prepared via an emulsion route; more specifically, melt-emulsification was carried out at temperatures above the lipids' melting points followed by quench cooling to form crystalline particles. The preparation process is fully described elsewhere. ${ }^{21}$ Systems were then stored at $4{ }^{\circ} \mathrm{C}$ until further analysis.

Emulsions were fabricated using two emulsification techniques at varying energy inputs. In particular, emulsions were produced using a high-shear mixer (Silverson L5 M, Silverson Machines Ltd, UK) with an emulsion screen of $19 \mathrm{~mm}$ diameter for 2 minutes at $9000 \mathrm{rpm}$, as well as an ultrasound generator for 30 seconds at 95\% amplitude (Sonics \& Materials, Inc., CT, USA). During both processes to avoid shear-induced heating of the sample, emulsification was carried out whilst the vessel was immersed in an ice bath. Phase inversion was tested by simple dilution to confirm that all emulsions remained water continuous. The resultant o/w emulsions were analysed directly post production or were stored at $4{ }^{\circ} \mathrm{C}$ until further analysis. 
Droplet size analysis. Stability against coalescence as a function of time was assessed by optical observation and droplet size measurements. Droplet size distributions for o/w emulsions were analysed by laser diffraction using a Malvern Mastersizer 2000S (Malvern Instruments, UK) equipped with a Hydro S dispersion cell. A refractive index of 1.47 (RI of the sunflower oil dispersed phase) was used for the calculation of the droplet size distribution. Samples were analysed immediately and after 7, 14 and 30 days following emulsion preparation.

Imaging emulsions microstructure by confocal laser light scanning microscopy (CLSM). Pickering performance (i.e. the location of the particles) was assessed visually using microscopy. Emulsion droplets were visualised at room temperature using a confocal laser light microscope, (Leica TCS SPE (Heidelberg, Germany)), equipped with laser operating at a wavelength of $532 \mathrm{~nm}$. The various emulsion components were individually stained at various times throughout production. Rhodamine B $(0.01 \mathrm{wt} / \mathrm{wt} \%)$ was added to the aqueous phase containing surface active species before mixing with the melted lipid. The solid lipid component was stained through addition of perylene $(0.01 \mathrm{wt} / \mathrm{wt} \%)$ to the hot $\mathrm{o} / \mathrm{w}$ emulsion following ultrasonication, prior to cooling. To image the oil phase Nile Red $(0.01 \mathrm{wt} / \mathrm{wt} \%)$ was added to the sunflower oil prior to emulsification. All the stains used were purchased from Sigma-Aldrich (SigmaAldrich, UK).

Differential scanning calorimetry (DSC) measurements. The melting properties of the emulsions were monitored at regular intervals by DSC using a high-sensitivity Setaram $\mu$ DSC7 evo microcalorimeter (Setaram Instrumentation, France). System equilibrium was achieved $\left(20^{\circ} \mathrm{C}\right)$ for $15 \mathrm{~min}$ prior to a heating ramp of $1{ }^{\circ} \mathrm{C} \min ^{-1}$ until a maximum temperature of $90{ }^{\circ} \mathrm{C}$ was reached. Within this temperature range the oil phase does not undergo any transition, thus, the endothermic peaks observed correspond to the melting of lipid crystalline particles present in the $\mathrm{o} / \mathrm{w}$ emulsions.

Thermal stability of emulsions. Adsorption of the lipid particles to the interface was studied by heating $\mathrm{o} / \mathrm{w}$ emulsions $(20 \mathrm{wt} / \mathrm{wt} \%)$ stabilised using cetyl palmitate particles $(2.5 \mathrm{wt} /$ $\mathrm{wt} \%)$ formed in the presence of sodium caseinate $(0.8 \mathrm{wt} /$ wt\%). The thermal behaviour of the lipid particles has been previously studied and it was demonstrated that it is highly dependent on the type and concentration of the surface active component used. ${ }^{21}$ More specifically, melting events commence at $c a .48 .2 \pm 0.2{ }^{\circ} \mathrm{C}$ with peak temperatures close to 50.7 $\pm 0.1^{\circ} \mathrm{C}$. A cross comparison between emulsion droplet sizes at temperatures both significantly lower and higher than the particle's melting point provides a better insight to the stabilising properties of the wax particles. As such, emulsions were placed in an oven (Advantage Lab, Belgium) at two preconditioned temperatures; 36 and $58{ }^{\circ} \mathrm{C}$ for 1 and 5 days. Changes in droplet size were monitored via laser diffraction measurements. Sodium azide (Sigma-Aldrich, UK) was added to all systems at a concentration of $0.001 \%(\mathrm{wt} / \mathrm{wt})$ to prevent microbiological growth.

\section{Results and discussion}

Emulsification was investigated in the presence of solid lipid particles formed with different surface active species (Tween 80 and $\mathrm{NaCas}$ ) and $\mathrm{o} / \mathrm{w}$ emulsions were prepared with sunflower oil as the dispersed phase. Emulsions were formed by using an ultrasonic probe and by a conventional high-shear mixing process. Preliminary studies showed that tristearin and cetyl palmitate particles fabricated in the absence of surface active species could not produce stable $\mathrm{o} / \mathrm{w}$ emulsions, on one hand due to their inherent hydrophobic character and on the other, due to their large particle sizes. As such, the emulsions assessed all contained lipid particles $(2.5 \mathrm{wt} / \mathrm{wt} \%$ of the aqueous phase) formed with either Tween 80 or NaCas (both at $0.8 \mathrm{wt} / \mathrm{wt} \%$ of the aqueous phase). The parameters studied were the lipid type that the particles were composed of, the type of surface active agents used in the production of the particles, as well as the mass fraction of sunflower oil.

\section{Effect of formulation parameters on emulsion droplet size}

The effect of the presence of particles formed with a different surface active component on the emulsion's droplet size and as a function of the oil's fraction is shown in Fig. 1. This was
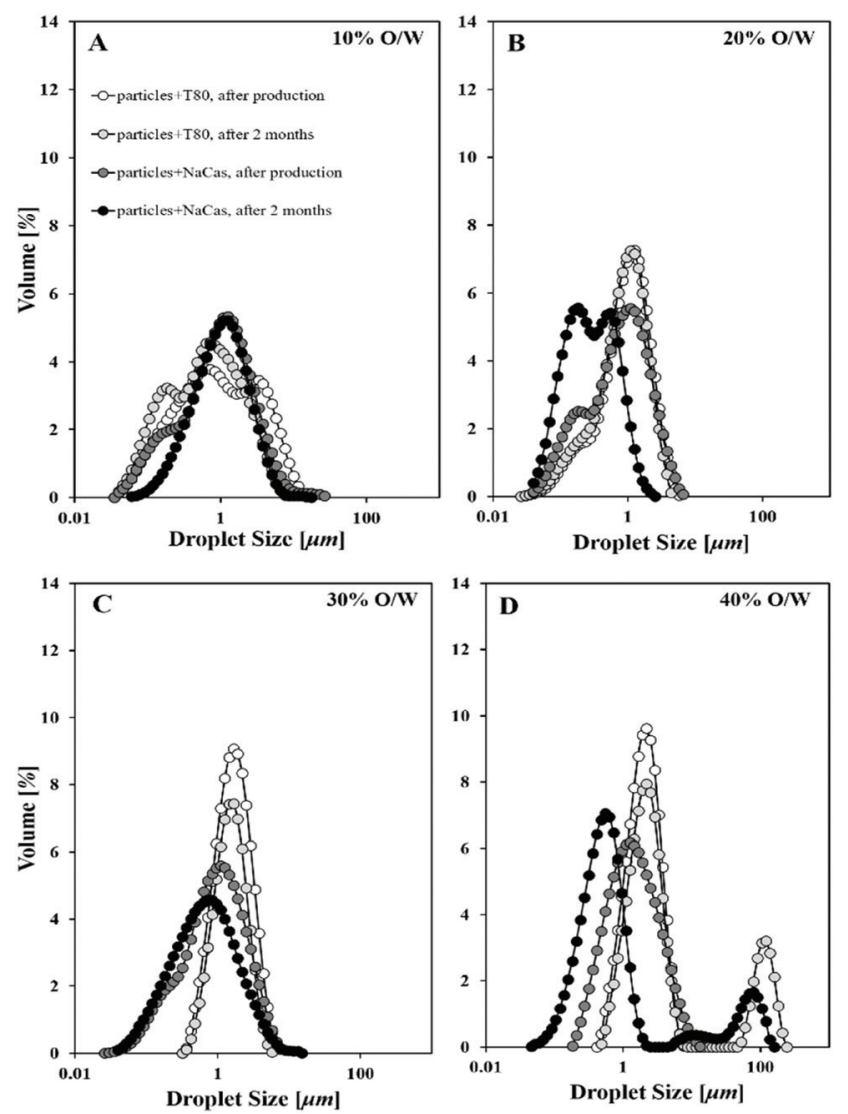

Fig. 1 Variations of emulsion droplet size as a function of dispersed phase mass fraction and different surface active species used in the preparation of solid wax particles. Cetyl palmitate and surface active species were used at a constant concentration of 2.5 and $0.8 \mathrm{wt} / \mathrm{wt} \%$ of the aqueous phase respectively across all samples. 
measured directly after emulsification, and also after two months of quiescent storage at refrigeration temperatures.

It can be seen that the average droplet size $\left(D_{4,3}\right)$ of all emulsions after ultrasound emulsification was $\sim 1-2 \mu \mathrm{m}$. Emulsion droplet size increased slightly with an increase in the internal phase mass fraction for lipid particles containing either of the two surface active species. It is anticipated that at a constant surface active component-to-oil ratio, an increase in the oil mass fraction engenders an enhanced rate of collisions and coalescence, with the coalescence rate increasing much faster than the rate of droplet break-up. ${ }^{24}$ This is common place for emulsions where Tween 80 or NaCas have been used as the sole emulsifiers and/or stabilisers. ${ }^{25}$

Additionally, when particles were formed with sodium caseinate the resulting emulsion had smaller average droplet sizes than when Tween 80 was used, and were also more stable over time, particularly for intermediate oil mass fractions (i.e. 20 and $30 \%$ ). This droplet size discrepancy is likely to originate from the lower levels of interfacial tension induced by Tween 80. This reduction in interfacial tension during initial droplet formation results in an increased number of small emulsion droplets (within a fixed volume) which, in turn, increases the probability of droplet-droplet collisions (during processing). ${ }^{26}$ Further, potential differences in the thickness of the interfacial film that particles with Tween 80 or NaCas would provide, could be accounted for the droplet size disparity observed; enhanced steric properties caused by the thicker protein-stabilised interface would form a barrier to droplet coalescence. ${ }^{8}$ Another factor that needs to be considered is the amount of free Tween 80 or NaCas (i.e. not associated with the lipid particles) in the original particle dispersions, which could also be having an impact, e.g. acting to adsorb both to the oil droplet and wax particle interface. In all cases, it has to be considered that during sonication, there's always the risk of jeopardising the integrity of solid matter due to local temperature increase.

Upon ageing, droplet coalescence was observed only for the highest dispersed phase content (i.e. 40\%) with a population appearing at around $100 \mu \mathrm{m}$ for emulsions stabilised by lipid particles containing either surface active species. It is proposed here that at an oil mass fraction of $40 \%$, instability becomes more prominent as a result of the loss of particulate matter, the mechanism of which will be the focus of a latter section. An enhanced desorption of solid lipid particles from the interface or dissolution is probably taking place, which leads to droplets having their surfaces devoid of stabilising material, therefore prone to inter-droplet contacts and subsequent coalescence. Similar observations were made by Gupta and Rousseau that used emulsion surface charge measurements and TEM images to explain the destabilisation process occurring in SLN-decorated $\mathrm{o} / \mathrm{w}$ emulsions. ${ }^{27}$ A significant drop of $10 \mathrm{mV}$ (emulsion surface charge) was recorded by 24 weeks, and non-covered emulsion interfaces together with SLN in the continuous phase were depicted in the micrographs, providing evidence of the gradual destabilisation of the emulsion.

\section{Effect of processing method on emulsion behaviour}

Emulsions were also produced using a conventional processing method that provides a lower level of shear in comparison to sonication, e.g. high-shear homogenisation. A study investigating differences between emulsions formed in the presence of cetyl palmitate or tristearin particles fabricated with low amounts of sodium caseinate was conducted. There was no control over instability due to creaming and all the obtained emulsions creamed within minutes following production. However, there was no oil layer observed with time; this would have indicated the occurrence of coalescence phenomena. The mean droplet sizes of these systems are illustrated in Fig. 2 for different storage times (at $4^{\circ} \mathrm{C}$ ).

It is evident from Fig. 2 that the use of ultrasound generates emulsions with droplets of about an order of magnitude lower than the ones produced via a high-shear device. It is known that ultrasound, due to cavitational collapse, promotes an enhanced droplet break-up, enabling the eventual formation of very fine/nano droplets. ${ }^{28-30}$ Moreover, the smaller sized
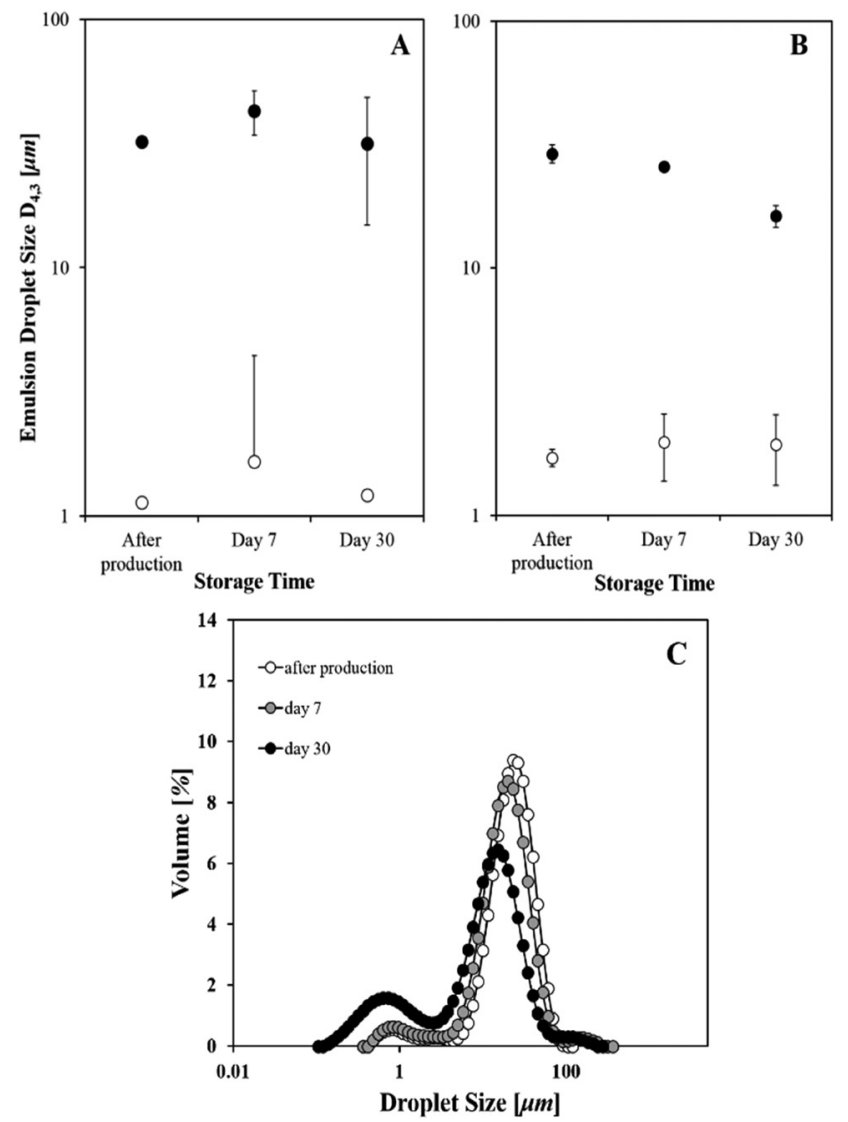

Fig. 2 Volume-weighted mean droplet diameters $\left(D_{4,3}, \mu \mathrm{m}\right)$ of $20 \% \mathrm{o} / \mathrm{w}$ emulsions formed with wax (A) and triglyceride (B) particles with $0.8 \%$ sodium caseinate, using different processing conditions, measured immediately after preparation and after 7 and 30 days. Closed and open circle symbols refer to emulsions prepared by high-shear mixer and ultrasound respectively. (C) Droplet size distributions of the systems presented in (B) using high-shear mixing. Where not visible error bars are smaller than the symbols. 
emulsions were also more stable over the investigated time, yet this is also true for the emulsions with cetyl palmitate particles produced with a high-shear homogeniser (Fig. 2A). The initial droplet size does not appear to depend on the lipid source of the particles, however the long-term emulsion stability was more sensitive to that parameter. The $D_{4,3}$ value of the emulsions with tristearin particles and NaCas manufactured by a high-shear mixer, drops from 29.1 to $16.2 \mu \mathrm{m}$ following one month storage. The relatively broad distribution was composed of two populations, one at $\sim 1 \mu \mathrm{m}$ and a larger one at $\sim 23 \mu \mathrm{m}$ (Fig. 2C). The smaller peak is likely to correspond to lipid particles that are not associated with the oil droplets ("free"). Over time, this peak appears to grow at the expense of the bigger one, potentially pointing to increased desorption of particles from the interface. This shift in the distribution is what also causes the subtle drop in $D_{4,3}$ values as seen in Fig. 2B. In regards to emulsions produced in the presence of cetyl palmitate particles formed with Tween 80, emulsification was not possible under high-shear mixing.

In order to confirm that emulsion stabilisation was imparted by the lipid particles rather than the surface active agents used for their fabrication, emulsion's microstructure was visualised. Most commonly, cryo-SEM has been used for visualisation of emulsion microstructure and for lipid crystals residing at the interface in particular. ${ }^{15,31,32}$ In addition, confocal laser light microscopy ${ }^{33}$ and $\mathrm{TEM}^{27}$ have been employed for investigation of the ultra-structure of o/w emulsions decorated by solid lipid nanoparticles.

Fig. 3A compares droplet size distributions of emulsions prepared with particles and sodium caseinate and those ones stabilised by protein only. Fig. 3B displays a CLSM image of a $20 \% \mathrm{o} / \mathrm{w}$ emulsion prepared in the presence of the same species and produced with a high shear mixer, taken within two hours after its production.
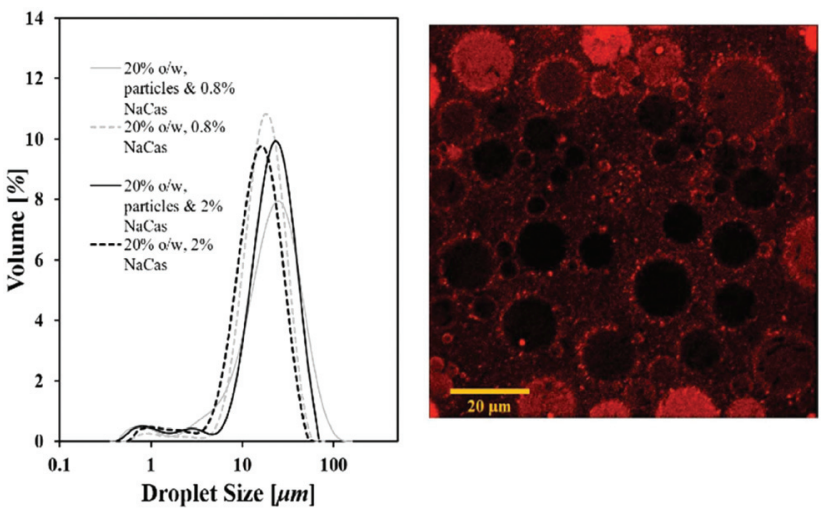

Fig. 3 Droplet size distribution of $20 \%$ sunflower-in-water emulsions containing solid wax nanoparticles formed in the presence of sodium caseinate $(0.8,2 \%)$ or stabilised by the same concentration of neat sodium caseinate (A). Measurements were conducted immediately after production. Representative confocal image of the emulsion stabilised by wax particles with $\mathrm{NaCas}(\mathrm{B})$. The micrograph was taken within 2 hours following emulsion production.
It can be seen from Fig. 3A that the droplet size of lipid particle-stabilised emulsions is not significantly different to protein-stabilised systems. Any shift can be attributed to the different level of interfacial tension reduction that each of the species provides. A closer view to emulsions' microstructure obtained via confocal microscopy suggests the presence of a close association of lipid particles with the interfacial area of the emulsion (Fig. 3B). It appears that wax particles reside at the space around the oil droplets and are adsorbing at the interface. This data provides evidence of a Pickering-type stabilisation with solid lipid particles - rather than the surface active agents utilised for their fabrication - acting as emulsion stabilisers. The demonstrated mechanism is also in agreement with a previous study by Pawlik et al. who confirmed a Pickering performance of tripalmitin particles fabricated with whey protein isolate (WPI) in the stabilisation of sunflower-inwater emulsions. ${ }^{15}$

\section{Effect of dispersed oil phase type on Pickering emulsion formation and stability}

The effect of the dispersed phase polarity on the resulting emulsion droplet size and stability was studied by using pure alkanes of different molecular sizes (chain length). Fig. 4 presents laser diffraction measurements performed on dodecane and hexadecane-in-water emulsions where the aqueous phase contained wax particles formed with Tween 80. Emulsion stability was monitored over 10 and 75 days of storage (at $\left.4^{\circ} \mathrm{C}\right)$.

It was anticipated that altering the polarity of the oil phase would impact solid particles' surface characteristics, i.e. how particles adsorb and reside at an emulsion droplet interface. In general, oil type is one of the parameters that influence interfacial tension, solids' contact angle, as well as the energy
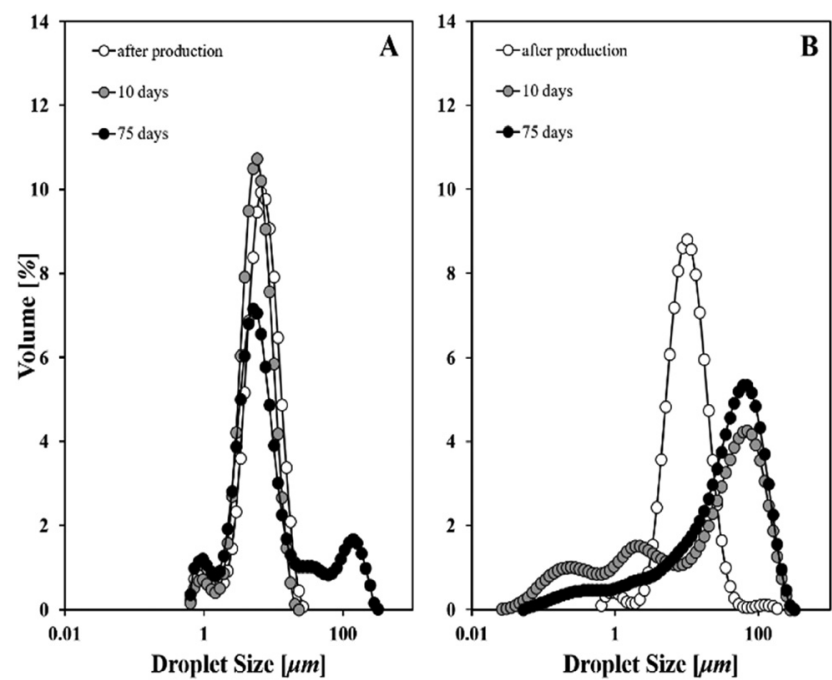

Fig. 4 Droplet size distributions of $20 \%$ dodecane (A) and hexadecane (B) in-water emulsions stabilised by $2.5 \mathrm{wt} / \mathrm{wt} \%$ wax particles formed with $0.8 \mathrm{wt} / \mathrm{wt} \%$ Tween 80 . Measurements were performed immediately after production and following 10 and 75 days of cold storage. 
of particle anchoring to an interface. ${ }^{34}$ The order of increased polarity of the oils investigated in the current study is hexadecane $<$ dodecane $<$ sunflower oil. ${ }^{35}$ Comparing the droplet sizes of the emulsions produced with apolar oils, it is evident that larger sizes were yielded in the presence of a less polar oil (Fig. 4B). Previous work on the determination of interfacial tension for $n$-alkane/water systems has revealed a linear dependence between the carbon atoms in the molecule and interfacial tension. ${ }^{36,37}$ The authors of both studies reported that as the number of carbon atoms increases, interfacial tension increases accordingly. As such, when dodecane is used as the oil phase it enables a more effective lowering of the oil-water interfacial tension and therefore, droplets fragmentation is enhanced which, in turn, results in smaller sizes. We therefore postulate that interfacial properties are altered due to the presence of less polar oils, and this enables the formation of $\mathrm{o} / \mathrm{w}$ emulsions with droplet sizes that depend on the polarity of the dispersed phase.

These emulsions also appeared to be more prone to instability mechanisms over time, as the distribution was shifted to larger sizes following 10 days of cold storage pointing to coalescence (Fig. 4B). Earlier studies of Davis and Smith demonstrated a decreased emulsion stability (due to droplet coalescence) upon increase of oil phase's polarity. ${ }^{38}$ The emulsions in that case were stabilised solely by a surfactant (i.e. sodium dodecyl sulfate (SDS)) and different aliphatic hydrocarbons $\left(\mathrm{C}_{6}-\mathrm{C}_{16}\right)$ as dispersed phase oils were screened. More relevant to particle-stabilised emulsions, studies on model silica systems of similar hydrophobicities demonstrated an oiltype dependence of emulsion's continuous phase; particles within non-polar oils preferentially form o/w emulsions, whereas polar oils tend to produce w/o emulsions. ${ }^{39}$ This behaviour was explained based on the work of adhesion between water and oil and the contact angle between solid-oil-water (i.e. particles' hydrophobicity increased at polar interfaces). In the present study, it is expected that wax particles have different solubility in dodecane and hexadecane, and this is likely to largely explain the long-term stability of the produced emulsions. Another possibility is that particles are not well trapped by the interfaces between water and oil and this appears to be a function of oil's polarity.

\section{Stability of solid lipid particles within sunflower oil emulsions}

Dissolution of solid lipid particles within the disperse phase is anticipated to occur upon introduction of the oil phase, emulsification and upon storage. The migration of lipid molecules (from the solid lipid particle to the dispersed phase) will be driven by the solubility of the two species. ${ }^{20}$ Microcalorimetry was employed as the technique to track the evolution of the solid fat content in $\mathrm{o} / \mathrm{w}$ emulsions formed in the presence of $2.5 \mathrm{wt} / \mathrm{wt} \%$ wax particles and $0.8 \mathrm{wt} / \mathrm{wt} \%$ surface active species for different oil phase fractions. The melting thermograms of these emulsions are shown in Fig. 5A and B. Their melting enthalpies are expressed as a ratio $\Delta H_{\text {emulsion }} / \Delta H_{\text {dispersion }}$ and are plotted versus the disperse phase mass fraction (Fig. 5C and $\mathrm{D})$. Each of these $\Delta H$ values corresponds to the single
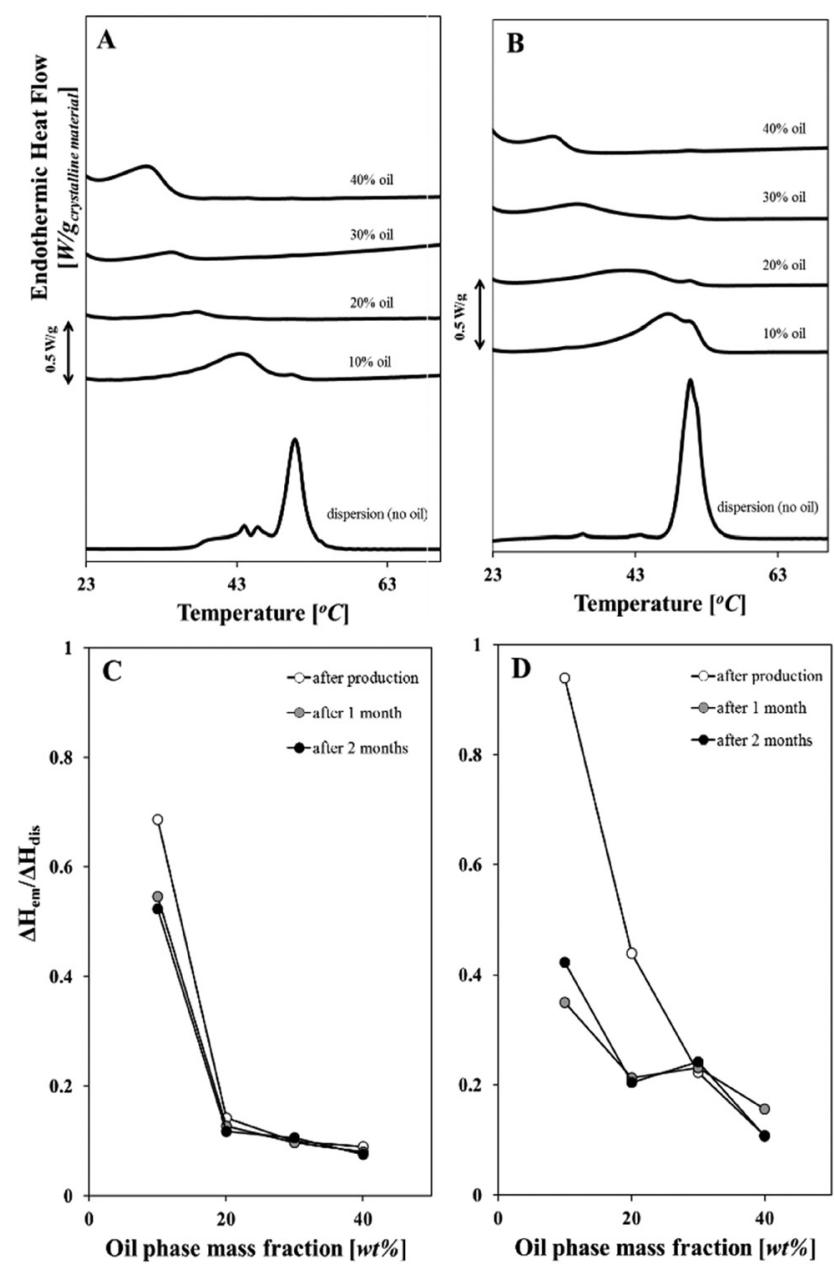

Fig. 5 Melting thermograms immediately after production of 10, 20, 30 and $40 \% \mathrm{o} / \mathrm{w}$ emulsions produced via sonication for $30 \mathrm{~s}(\mathrm{~A}, \mathrm{~B})$. Emulsions were produced using $2.5 \mathrm{wt} / \mathrm{wt} \%$ wax particles and $0.8 \mathrm{wt} /$ wt\% Tween 80 (A) or NaCas (B). The "dispersion" sample is the aqueous dispersion of wax particles formed in the presence of surface active species which is the continuous phase of all the emulsions. Time evolution of the amount of crystalline material present within emulsions $\left(\Delta H_{\mathrm{em}} / \Delta H_{\mathrm{dis}}\right)$ stabilised by lipid particles and Tween 80 (C) or NaCas (D) for different oil percentages. Results shown are from a representative experiment.

thermograms presented in Fig. 5A and B. The above mentioned ratio is used in order to denote the fraction of the solid matter that remains in the emulsion system following introduction of a liquid oil phase and subsequent emulsification, and along different storage times (at refrigeration temperatures). As such, a value of 1 would obviously suggest that the solid content is maintained, whereas a value that approximates zero would mean that any solid matter has been lost.

Dispersions of solid wax particles fabricated with Tween 80 or NaCas melted at approximately the same temperature $\left(\sim 50.5^{\circ} \mathrm{C}\right.$ is the main melting peak that corresponds to $\beta$-form crystals of cetyl palmitate), regardless of surface active species type. When the dispersion was mixed with sunflower oil to form the emulsion, the respective melting endotherm of the 
resulting emulsion occurred progressively at lower temperatures, as the dispersed phase (oil) mass fraction in the emulsion was increased (Fig. 5A and B). The magnitude of the change in the endothermic transition, as also depicted from the enthalpy ratio values (Fig. 5C and D), was significantly greater when the oil mass fraction was high (i.e. $40 \%)$. This indicates that solid matter suffers a greater loss $\left(\Delta H_{\mathrm{em}} / \Delta H_{\mathrm{dis}}<\right.$ 0.2 ) with an increase in the dispersed phase fraction, and this appears to be independent of the type of the surface active component. The results are well aligned with a study by Samtlebe et al. who investigated $n$-tetradecane emulsions in the presence of crystalline $n$-eicosane particles..$^{20}$ The authors observed a marked difference in the melting thermograms of 9:1 and 1:9 mixtures of $n$-eicosane SLN and $n$-tetradecane emulsions after different storage times. They ascribed the distinct melting profiles to changes in solid fat content, as measured by nuclear magnetic resonance spectroscopy (NMR).

In the case of emulsions stabilised by wax particles and Tween 80 the enthalpy ratio decreased sharply from 0.68 to 0.14 with an increase from a 10 to a $20 \%$ mass fraction of sunflower oil in the system and decreased only marginally for higher oil contents (Fig. 5C). In earlier studies for $n$-alkane droplets in the presence of a non-ionic surfactant, it has been observed that oil exchange takes place between droplets and the rate of this exchange increases in a linear fashion with the droplet surface area. ${ }^{40}$ In the current study, a high rate of oil transfer would essentially mean increased dissolution, hence, to an extent, the preceding observation is true as well. It is also worth noting that in the emulsion containing $40 \%$ oil, the dissolution of particles is so significant that only approximately $9 \%$ of the initial lipid crystals (contained in the initial aqueous dispersion) were present in the emulsion following its production. While the emulsion with a $10 \%$ oil phase fraction suffered an almost $24 \%$ decrease in the amount of solid lipids, the rest of the fractions appeared to have negligible changes over the course of two months. This clearly suggests that loss of solid matter occurs shortly following emulsification, and prolonged storage does not substantially result in any further losses.

In contrast, the melting enthalpies ratios for the emulsions stabilised by wax particles with sodium caseinate were higher (as absolute values) (Fig. 5D) than the respective ones for wax particles in the presence of Tween 80. This behaviour persisted across all investigated mass fractions, except the $40 \%$ where the two systems display similar levels of solid matter losses (Fig. 5C and D). Counter to the system in the presence of Tween 80 , sodium caseinate doesn't give a plateau behaviour at oil mass fractions $\geq 20 \%$ and an increase in the dispersed phase mass fraction produces a further drop in the remaining solid material.

A number of studies has shown that the rate of oil exchange between $\mathrm{o} / \mathrm{w}$ emulsion droplets is highly dependent on the type and concentration of the surfactant present. ${ }^{19,40-42}$ More specifically, McClements and Dungan demonstrated that solubilisation of hydrophobic molecules and subsequent transport between oil droplets is promoted by surfactant micelles. ${ }^{40}$ The data presented above provide evidence of solubilisation of hydrophobic material from the SLN and potential transfer into the oil droplets. This is promoted by increases in the mass fraction of the oil phase and it is also facilitated by the surface active species present, most likely by its ability to form micelles and assist transportation from SLN to the oil phase.

Based on evidence in literature, the rate of SLN dissolution is also affected by the lipid source, e.g. its molecular weight. It has been found that the rate of oil mass transfer in emulsions decreases with an increase in lipid's molecular weight. ${ }^{20}$ Therefore, the loss of solid matter in the presence of sodium caseinate was further investigated on whether it can be enhanced or maintained by changing the source of lipid particles to a lipid of a higher molecular weight (hence, reduced solubility). Oil-in-water emulsions were prepared (via ultrasound) using tristearin particles formed with sodium caseinate and their melting enthalpy ratios were juxtaposed to the ones obtained from the respective ones of emulsions with wax particles. These ratios are presented in Fig. $6 \mathrm{~A}$ along with the emulsions' droplet size evolution for each investigated oil mass fraction (Fig. 6B and C).
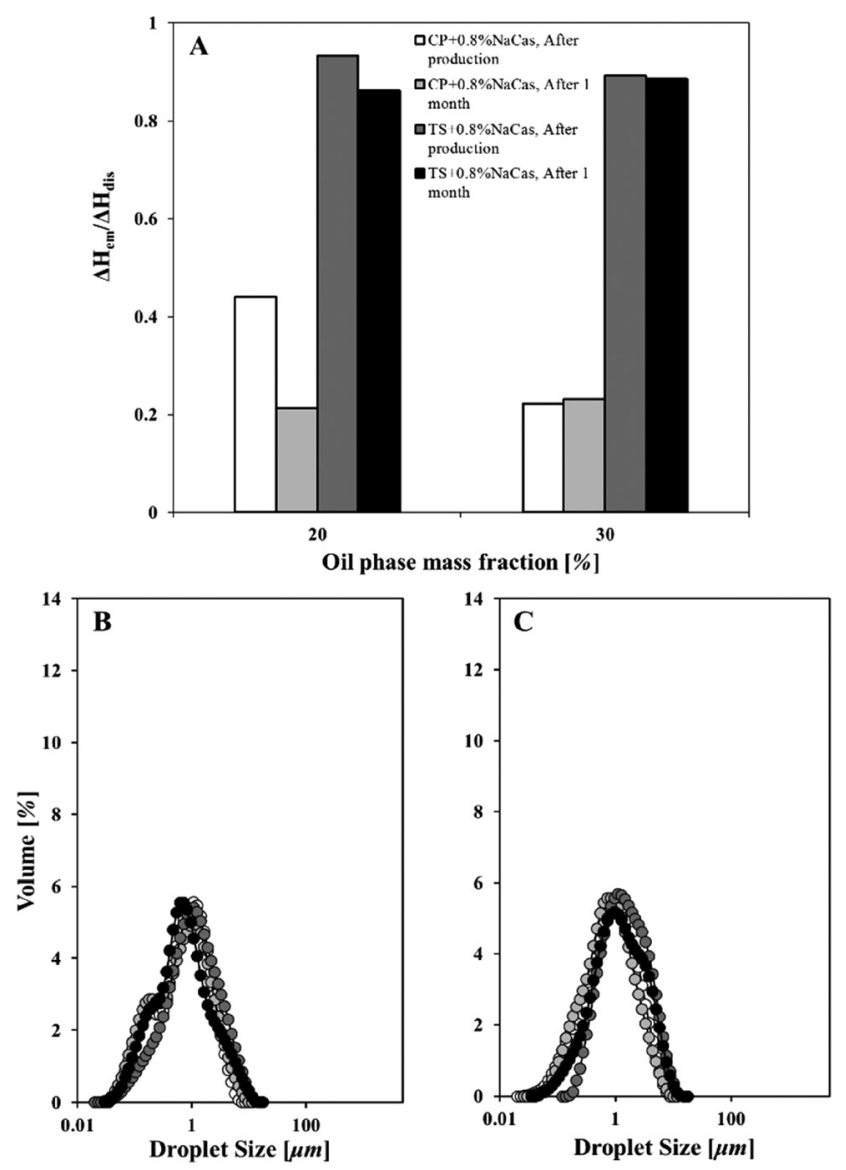

Fig. 6 Time evolution of the ratio $\Delta H_{\mathrm{em}} / \Delta H_{\text {disp }}$ for emulsions stabilised by cetyl palmitate and tristearin particles, both formed in the presence of $0.8 \% \mathrm{NaCas}(\mathrm{A})$. Droplet size distributions of the respective emulsions at $20 \%(B)$ and $30 \%$ (C) dispersed phase mass fraction. 
As depicted from Fig. 6A, dissolution of solid tristearin particles in sunflower emulsions was significantly suppressed. There was no evidence of substantial loss of solid matter in the time allowed and that appeared to be independent of dispersed phase concentration. This indicates that the amount of tristearin particles when fabricated using sodium caseinate is negligibly affected by the presence of liquid oil. A percentage as high as $87 \%$ of the original crystals is still available after one month storage to stabilise the oil droplets.

One would expect that the almost $52 \%$ loss of solid content in the case of wax particles (Fig. 6A) following one month storage (at $20 \%$ oil mass fraction) would have an impact on their functionality as emulsion stabilisers. The fact that no differences were detected in the droplet size patterns within a month (Fig. 6B) potentially points to a sufficient amount of particles being available in the first place to stabilise the interfaces.

\section{Thermal stability of emulsions stabilised by solid lipid particles}

This section is looking at the thermal stability of the emulsions' microstructure in an attempt to investigate the link to temperature effects on the lipid particles themselves. For this purpose, o/w emulsions stabilised by cetyl palmitate particles that have been formed in the presence of $0.8 \%$ NaCas were stored at elevated temperatures; at a temperature well below the melting point of the lipid particles (i.e. $36^{\circ} \mathrm{C}$ ) and at a temperature that the crystalline material is already melted (i.e. $\left.58{ }^{\circ} \mathrm{C}\right)$. Fig. 7 depicts the droplet size profiles obtained upon exposure of emulsions to increased temperatures and also upon ageing.

Laser diffraction measurements showed that subjection to elevated temperatures (either 36 or $58^{\circ} \mathrm{C}$ ) resulted in the formation of additional size populations and a shift towards higher measured emulsion diameters (Fig. 7A and B). This
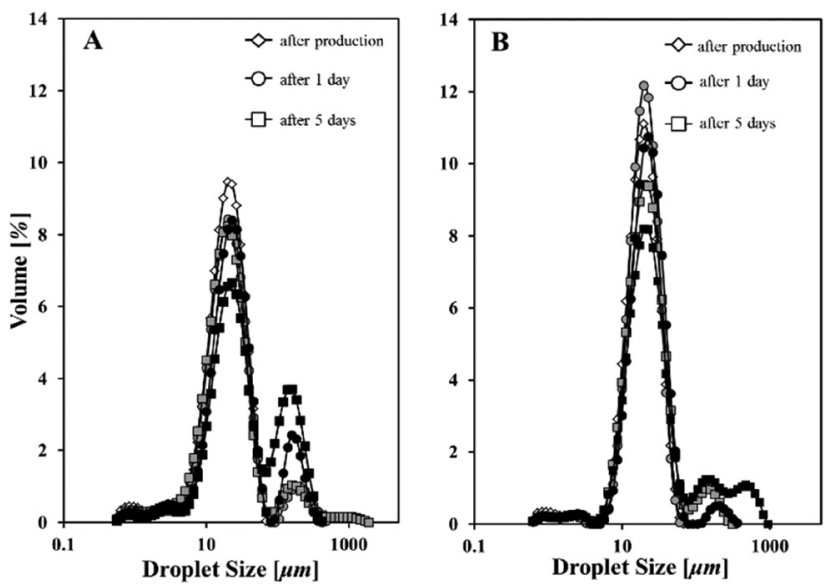

Fig. 7 Comparative size distributions of solid wax particles and $\mathrm{NaCas}$ (0.8\%) stabilised emulsions with $20 \%$ (A) and $40 \%$ (B) sunflower oil. Open symbols represent emulsions after production, while coloured symbols show emulsions stored at elevated temperatures; grey and black colour symbols represent emulsions stored at $36^{\circ} \mathrm{C}$ and $58^{\circ} \mathrm{C}$ respectively. evidence of coalescence occurring in the systems regardless of the dispersed phase mass fraction, appears to be more accentuated at temperatures above cetyl palmitate's melting point (i.e. $55{ }^{\circ} \mathrm{C}$ ) rather than at $36^{\circ} \mathrm{C}$. Stability of the emulsion systems clearly depends on the thermal properties and this has come about due to the presence of lipid particles themselves.

\section{Conclusions}

This study demonstrated the potential of solid lipid particles of colloidal dimensions to act as Pickering stabilisers of oil-inwater emulsions. Particles that were fabricated in the presence of minimal amounts of a surface active component could form stable to coalescence o/w emulsions for at least two months. Emulsion stability was corroborated to dispersed phase mass fraction and polarity, processing method employed, as well as inherent thermal properties of the stabilising lipid particles. Dissolution of the solid matter upon introduction of liquid sunflower oil, subsequent emulsification and over a storage period was shown to take place and was monitored via DSC. This phenomenon was more significant for the smaller lipid molecule (e.g. cetyl palmitate) as opposed to the long-chained tristearin. Dissolution was also more pronounced in the presence of a surface active species that is capable of forming micelles (i.e. Tween 80). Despite the loss of crystalline material from the interface there was no concomitant compromise in particles' Pickering functionality and emulsions remained stable for one month. This was ascribed to a sufficient amount of solid lipid particles being present, although screening of different concentrations of lipid particles would provide a deeper insight into the interplay between particle content/ interfacial coverage and emulsion stability.

Taking this work further, it would be interesting to see how the surface active components' content, irrespective of particle size, affects particles' Pickering performance with the ultimate goal being Pickering stabilisation only in the presence of colloidal lipid structures. The amount of surface active species could be removed via a range of purification/washing procedures (e.g. ultracentrifugation, diffusion through dialysis tubing, cross flow filtration, etc.). ${ }^{43}$ Last but not least, assessment of longer storage stability of emulsions would certainly be more relevant to commercial applications.

\section{Acknowledgements}

The authors gratefully acknowledge financial support from EPSRC, Innovate UK and Cargill. We thank Dr Richard L. Watson for helping with the confocal equipment and software.

\section{References}

1 D. J. McClements, Food Emulsions: Principles, Practices and Techniques, CRC Press, Florida, 2016. 
2 V. J. Morris, Trends Biotechnol., 2011, 29, 509-516.

3 D. J. French, A. T. Brown, A. B. Schofield, J. Fowler, P. Taylor and P. S. Clegg, Sci. Rep., 2016, 6, 31401.

4 I. Odriozola-Serrano, G. Oms-Oliu and O. Martín-Belloso, Front. Nutr., 2014, 1, 24.

5 R. Pichot, F. Spyropoulos and I. T. Norton, J. Colloid Interface Sci., 2009, 329, 284-291.

6 E. Dickinson, Curr. Opin. Colloid Interface Sci., 2010, 15, 40-49.

7 M. Kargar, K. Fayazmanesh, M. Alavi, F. Spyropoulos and I. T. Norton, J. Colloid Interface Sci., 2012, 366, 209-215.

8 C. C. Berton-Carabin, M.-H. Ropers and C. Genot, Compr. Rev. Food Sci. Food Saf., 2014, 13, 945-977.

9 N. Niknafs, Doctor of Philosophy, University of Birmingham, 2011.

10 L. L. Lee, N. Niknafs, R. D. Hancocks and I. T. Norton, Trends Food Sci. Technol., 2013, 31, 72-78.

11 S. Frasch-Melnik, I. T. Norton and F. Spyropoulos, J. Food Eng., 2010, 98, 437-442.

12 S. Ghosh, T. Tran and D. Rousseau, Langmuir, 2011, 27, 6589-6597.

13 D. Rousseau, Curr. Opin. Colloid Interface Sci., 2013, 18, 283-291.

14 N. Garti, H. Binyamin and A. Aserin, J. Am. Oil Chem. Soc., 1998, 75, 1825-1831.

15 A. Pawlik, D. Kurukji, I. Norton and F. Spyropoulos, Food Funct., 2016, 7, 2712-2721.

16 J. N. Coupland, J. Weiss, A. Lovy and D. J. McClements, J. Food Sci., 1996, 61, 1114-1117.

17 J. N. Coupland, D. Brathwaite, P. Fairley and D. J. McClements, J. Colloid Interface Sci., 1997, 190, 71-75.

18 J. Weiss, J. N. Coupland, D. Brathwaite and D. J. McClements, Colloids Surf., A, 1997, 121, 53-60.

19 J. Weiss and D. J. McClements, Langmuir, 2000, 16, 58795883.

20 M. Samtlebe, U. Yucel, J. Weiss and J. N. Coupland, J. Am. Oil Chem. Soc., 2012, 89, 609-617.

21 I. Zafeiri, J. E. Norton, P. Smith, I. T. Norton and F. Spyropoulos, J. Colloid Interface Sci., 2017, 500, 228240.

22 T. Prevc, B. Cigić, R. Vidrih, N. Poklar Ulrih and N. Šegatin, J. Agric. Food Chem., 2013, 61, 11355-11362.

23 M. Montal and P. Mueller, Proc. Natl. Acad. Sci. U. S. A., 1972, 69, 3561-3566.
24 A. Hakansson, in Engineering aspects of food emulsification and homogenization, ed. M. Rayner and P. Dejmek, CRC Press, Florida, 2015, pp. 125-148.

25 C. Sun and S. Gunasekaran, Food Hydrocolloids, 2009, 23, 165-174.

26 P. Y. Liu, R. Y. Yang and A. B. Yu, Phys. Fluids, 2011, 23, 013304.

27 R. Gupta and D. Rousseau, Food Funct., 2012, 3, 302-311.

28 J. P. Canselier, H. Delmas, A. M. Wilhelm and B. Abismaïl, J. Dispersion Sci. Technol., 2002, 23, 333-349.

29 S. Kentish, T. J. Wooster, M. Ashokkumar, S. Balachandran, R. Mawson and L. Simons, Innovative Food Sci. Emerging Technol., 2008, 9, 170-175.

30 T. S. H. Leong, T. J. Wooster, S. E. Kentish and M. Ashokkumar, Ultrason. Sonochem., 2009, 16, 721-727.

31 S. Frasch-Melnik, F. Spyropoulos and I. T. Norton, J. Colloid Interface Sci., 2010, 350, 178-185.

32 D. Kurukji, R. Pichot, F. Spyropoulos and I. T. Norton, J. Colloid Interface Sci., 2013, 409, 88-97.

33 J. Heisler, K. Oehlke, R. Greiner and A. Steffen-Heins, presented in part at the InsideFood Symposium,Leuven, Belgium, 2013.

34 R. J. G. Lopetinsky, J. H. Masliyah and Z. Xu, in Colloidal Particles at Liquid Interfaces, ed. B. P. Binks and T. S. Horozov, Cambridge University Press, Cambridge, 2006, pp. 186-224.

35 V. Rampon, C. Brossard, N. Mouhous-Riou, B. T. Bousseau, G. Llamas and C. Genot, Adv. Colloid Interface Sci., 2004, 108-109, 87-94.

36 S. Zeppieri, J. Rodríguez and A. L. López de Ramos, J. Chem. Eng. Data, 2001, 46, 1086-1088.

37 R. Aveyard and D. A. Haydon, Trans. Faraday Soc., 1965, 61, 2255-2261.

38 S. S. Davis and A. Smith, in Theory and practice of emulsion technology, ed. A. L. Smith, Academic Press Inc., London, 1976.

39 B. P. Binks and S. O. Lumsdon, Phys. Chem. Chem. Phys., 2000, 2, 2959-2967.

40 D. J. McClements and S. R. Dungan, J. Phys. Chem., 1993, 97, 7304-7308.

41 D. J. McClements, S. R. Dungan, J. B. German and J. E. Kinsella, Colloids Surf., A, 1993, 81, 203-210.

42 D. J. McClements, S. R. Dungan, J. B. German and J. E. Kinsella, J. Colloid Interface Sci., 1993, 156, 425-429.

43 J. Veeken, IOP Conf. Ser.: Mater. Sci. Eng., 2012, 40, 012035. 